\title{
KM-Finder: Uma Ferramenta para Detecção de Motivos
}

\author{
Luciana Montera $^{1}$, Lucas Akayama Vilhagra $^{1}$, Tainá Raiol ${ }^{2}$ \\ ${ }^{1}$ Faculdade de Computação - Universidade Federal de Mato Grosso do Sul (UFMS) \\ Caixa Postal 549. CEP 79070-900. Campo Grande - MS - Brasil \\ ${ }^{2}$ Instituto Leônidas e Maria Deane - Fundação Oswaldo Cruz \\ Manaus - AM - Brasil \\ montera@facom.ufms.br, akayamalucasl@gmail.com, tainaraiol@amazonia.fiocruz.br
}

\begin{abstract}
The identification of patterns within specific regions of the HPV genome may contribute to the understanding of the viral pathogenesis. In this work, a tool for searching nucleotide patterns (motifs) in one or a group of DNA sequences was developed, which continues to be a computational challenge.
\end{abstract}

Resumo. A identificação de padrões em regiões específicas nos genomas de HPV pode contribuir para o entendimento da patogênese viral. Neste trabalho, foi desenvolvida uma ferramenta para busca por padrões de nucleotídeos (motivos) em uma ou grupos de sequências de DNA, tarefa que continua a ser um desafio computacional.

\section{Introdução}

Os papilomavírus humanos (HPV), fatores etiológicos do câncer do colo do útero, são classificados em cutâneos ou mucosotrópicos. O genoma viral codifica proteínas estruturais necessárias à replicação e proteínas codificadas por oncogenes, que estão diretamente associadas com o desenvolvimento de câncer. O genoma viral possui cerca de 7.900 pares de bases (pb), sendo dividido em 8 genes codificadores de proteínas (L1, L2, E1, E2, E4, E5, E6 e E7) e duas regiões não codificadoras: NCR (Noncoding Region) e LCR, que se localiza entre L1 e E6, e possui 883 pb. A expressão dos oncogenes virais é controlada pela LCR (Long Control Region), que apresenta diversos sítios de ligação para fatores transcricionais celulares e virais [Bernard 2013]. Acredita-se que o enhancer epitélio específico, localizado na LCR, contribui para o tropismo celular, propriedade importante na patogênese viral.

O objetivo deste trabalho é a identificação de padrões de nucleotídeos nas LCRs de HPV de um mesmo grupo e, possivelmente, inter-grupos que podem estar relacionados ao tropismo viral. Para isso, foi desenvolvida uma ferramenta de busca e outras duas ferramentas online foram utilizadas para comparação. Estão disponíveis 26 genótipos de genomas de HPV cutâneo e 13 de HPV mucosotrópico.

\section{Fundamentação Teórica}

Motivos são pequenas sequências que se repetem ao longo de uma molécula de DNA (ou entre moléculas distintas de DNA) as quais presume-se que tenham alguma função biológica [D'haeseleer 2006]. [Rajasekaran 2005] define três versões para o problema da busca por motivos em sequências biológicas: 
Definição 1 Planted $(l, d)$-Motif Search Problem - Dadas n sequências de mesmo tamanho e dois inteiro l e d, o problema consiste em encontrar o motivo de maior score de tamanho $l$ presente em todas as sequências $n$. Variações do motivo também são retornadas desde que difiram do motivo por uma distância de Hamming [Hamming 1950] não superior a $d$.

Definição 2 Edited Motif Search Problem - Dadas n sequências e três inteiros l, de q, são buscados todos os padrões das sequências de entrada de tamanho l que ocorram em pelo menos q das $n$ sequências. Um padrão $U$ é considerado uma ocorrência de outro padrão $V$ se a distância de edição [Levenshtein 1966] entre eles é no máximo d.

Definição 3 Simple Motif Search Problem - Dadas n sequências e um inteiro l, padrões definidos como uma string de símbolos juntamente com o caracter "?"(curinga) são buscados nas sequências. Um padrão não começa ou termina com "?”. O objetivo é identificar todos os padrões de tamanho máximo l, com número de curingas podendo variar de 0 a $\left\lfloor\frac{l}{2}\right\rfloor$, bem como o número de ocorrências destes padrões.

$\mathrm{Na}$ literatura existem diversos algoritmos de busca por motivos, dentre eles [Pevzner and Sze 2000], [Buhler and Tompa 2001], [Adebiyi and Kaufmann 2002] e [Dinh et al. 2012].

\section{Ferramentas e Resultados}

Ferramentas online, tais como SMILE [Marsan and Sagot 2000] e MEME [Bailey and Elkan 1994] realizam a busca por motivos em sequências biológicas. Neste trabalho, SMILE$^{1}$ e MEME $^{2}$, bem como uma ferramenta própria ${ }^{3}$ denominada KM-Finder ( $K$-mer and Motif Finder) foram utilizadas para buscar por padrões nas LCRs de HPV.

SMILE implementa um algoritmo combinatório baseado na construção de uma árvore de sufixo generalizada enquanto MEME [Bailey et al. 2009] é uma suíte de ferramentas que além de permitir realizar a busca por motivos permite a comparação de novos motivos encontrados com motivos já conhecidos e predição de função biológica.

A implementação proposta neste trabalho, KM-Finder, inicialmente determina grupos de substrings de tamanho $k$ ( $k$-mers), comparando cada $k$-mer e adicionando ao grupo os que possuem distância de Hamming de no máximo $m$ com relação ao $k$-mer representante. Os grupos que não tiverem ocorrências em no mínimo $q$ e no máximo $Q$ sequências do conjunto em estudo, são descartados. Para cada um destes grupos, são construídos motif-profiles, que são matrizes de frequências de cada nucleotídeo presente nos k-mers deste grupo. A concatenação dos nucleotídeos mais frequentes resulta em um candidato a motivo [Jones and Pevzner 2004]. A qualidade desse candidato é calculada fazendo-se a média aritmética dos valores dos nucleotídeos mais frequentes e, somente os motivos que tiverem qualidade superior a $p(0<p \leq 1)$ serão mostrados na saída. Para o motif-profile mostrado na Figura 1(b) a qualidade é 0.81 . A Figura 1 mostra como o motivo GCGACCGA foi determinado dado a busca no conjunto de LCR de mucosa com $k=8, q=13, Q=13, m=2$ e $p=0.8$. Na Figura 1(a) são mostrados 5 dos

\footnotetext{
${ }^{1}$ disponível em mobyle.pasteur.fr/cgi-bin/portal.py\#forms: :smile

${ }^{2}$ disponível em meme-suite.org/tools/meme

${ }^{3}$ disponível em pintado. facom.ufms.br/hpv
} 
23 elementos do grupo de $k$-mers utilizados para construir o motif-profile apresentado na Figura 1(b).

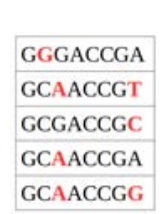

(a) Grupo de $k$-mers

\begin{tabular}{|c|c|c|c|c|c|c|c|c|}
\hline GCGACGA & Posição 1 & Posição 2 & Posição 3 & Posição 4 & Posição 5 & Posição 6 & Posição 7 & Posição 8 \\
\hline A & 0.04 & 0 & 0.39 & 0.87 & 0.04 & 0 & 0 & 0.43 \\
\hline C & 0.04 & 0.83 & 0.04 & 0.13 & 0.87 & 1 & 0 & 0.26 \\
\hline G & 0.91 & 0.17 & 0.57 & 0 & 0.09 & 0 & 1 & 0.13 \\
\hline T & 0 & 0 & 0 & 0 & 0 & 0 & 0 & 0.17 \\
\hline
\end{tabular}

(b) motif-profile

Figura 1. Grupo de $k$-mers e respectiva motif-profile que resulta no motivo GCGACCGA.

A fim de comparar as três ferramentas citadas, as buscas foram realizadas com parâmetros iguais ou similares. Os tamanhos de motivos buscados variaram entre 6 e 19. Para o grupo de HPV cutâneo na busca por motivos de tamanho 6, KM-Finder e SMILE encontraram a sequência AATAAA, enquanto MEME, além de encontrar essa sequência, encontrou também TGCCAA. Buscando por motivos de tamanho 19, KMFinder encontrou a sequência AGCGACCGATTTCGGTACC, enquanto MEME encontrou GATTGTTGCCAACAATCAT e SMILE retornou um resultado inesperado com 74.325 motivos. Para o grupo de HPV mucosotrópico, buscando motivos de tamanho 12, SMILE, KM-Finder e MEME encontraram a sequência ACCGATTTCGGT. SMILE encontrou ainda outras 8 sequências e MEME encontrou ACCGAAAACGGT e AACCGAAATCGG.

As divergências nos resultados vem do fato das ferramentas implementarem algoritmos diferentes, sendo assim, para uma mesma entrada, as saídas podem ser diferentes. Pode-se notar que quanto menos conservados são os motivos, mais os resultados apresentados pelas ferramentas divergem.

O tempo de resposta do MEME foi o melhor entre as ferramentas testadas e a apresentação dos resultados, por meio da construção de logos e outros gráficos, facilita a análise. SMILE responde rapidamente buscando motivos com tamanho até 16, porém, para tamanhos superiores, demora várias horas. Além da diferença no algoritmo implementado, um importante diferencial do KM-Finder é a possibilidade de busca simultânea em dois grupos distintos, pois, no caso do HPV, deseja-se observar possíveis semelhanças e diferenças entre cada grupo.

A Figura 2 apresenta o Diagrama de Venn resultante da busca por motivos para $k=9, m=2$ e $p=0.8$. Foram encontrados 603 motivos exclusivos de mucosa e 17 exclusivos de cutâneo enquanto que 9 motivos estão presentes em ambos os grupos. Padrões que caracterizem cada grupo de vírus podem ser utilizados como método de diagóstico para identificar a origem de determinado tumor, além de contribuir para o conhecimento sobre a patologia da doença.

\section{Conclusão e Trabalhos Futuros}

O prognóstico de uma neoplasia depende do tecido afetado que, no caso de tumores causados pela infecção por HPV, pode ter origem no epitélio ou mucosa. Neste trabalho, foi 


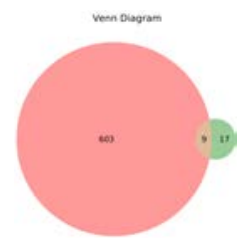

\section{Figura 2. Diagrama de Venn resultante da busca por motivos em sequências de HPV de mucosa (rosa) e cutâneo (verde).}

possível identificar padrões únicos que diferem entre os grupos de HPV, indicando potenciais marcadores para diagnóstico. Este estudo, ainda, gerou dados preliminares para a futura identificação de potenciais motivos envolvidos no tropismo viral. Para tal, existem ferramentas, como TFBind ${ }^{4}$, cujas análises podem ser incorporadas na ferramenta proposta. É possível ainda melhorar o algoritmo pela implementação de métodos mais sofisticados para mensurar a qualidade dos motivos a fim de tentar reduzir ainda mais falsos positivos e implementar outras representações gráficas para auxiliar no entendimento dos resultados.

\section{Referências}

Adebiyi, E. F. and Kaufmann, M. (2002). Extracting common motifs under the levenshtein measure: theory and experimentation. pages 140-156. Proc. Workshop on Algorithms for Bioinformatics (WABI).

Bailey, T. L., Boden, M., Buske, F., Frith, M., vGrant, C., and et al. (2009). Meme suite: tools for motif discovery and searching. pages 202-208. Nucleic Acids Research.

Bailey, T. L. and Elkan, C. (1994). Fitting a mixture model by expectation maximization to discover motifs in biopolymers. pages 28-36. Proceedings of the Second International Conference on Intelligent Systems for Molecular Biology.

Bernard, H.-U. (2013). Regulatory elements in the viral genome. Virology, 445(1):197-204.

Buhler, J. and Tompa, M. (2001). Finding motifs using random projections. pages 269-278. Proc. Fifth Annual International Conference on Computational Molecular Biology (RECOMB).

D'haeseleer, P. (2006). What are dna sequence motifs? pages 423-425. Nature Biotechnology.

Dinh, H., Rajasekaran, S., and Davila, J. (2012). qpms7: A fast algorithm for finding (1,d)-motifs in dna and protein sequences. In PLOS ONE.

Hamming, R. W. (1950). Error detecting and error correcting codes. The Bell System Technical Journal.

Jones, N. C. and Pevzner, P. A. (2004). An Introduction to Bioinformatics Algorithms. The MIT Press.

Levenshtein, V. I. (1966). Binary codes capable of correcting deletions, insertions and reversals. Soviet Physics-Doklady.

Marsan, L. and Sagot, M.-F. (2000). Algorithms for extracting structured motifs using a sufix tree with an application to promoter and regulatory site consensus identification. pages 345-362. Journal of Computational Biology.

Pevzner, P. and Sze, S. H. (2000). Combinatorial approaches to finding subtle signals in dna sequences. pages 269-278. Proc. Eighth International Conference on Intelligent Systems for Molecular Biology.

Rajasekaran, S. (2005). Algorithms for Motif Search in Handbook of Computational Molecular Biology. Chapman and Hall/CRC. chapter 37.

\footnotetext{
${ }^{4}$ disponível em t fbind.hgc.jp
} 